\title{
Improvement in Food Intolerance Symptoms after Pretreatment with Antibiotics Followed by Faecal Microbiota Transplantation: A Case Report
}

\author{
Annabel Clancy Thomas Borody \\ Centre for Digestive Diseases, Sydney, NSW, Australia
}

\section{Keywords}

Irritable bowel syndrome · Faecal microbiota transplantation · Food intolerance

\begin{abstract}
Irritable bowel syndrome (IBS) is a chronic gastrointestinal condition characterized by abdominal pain and altered bowel habits. Non-immune food reactions or food intolerances affect up to $20 \%$ of the general population and are commonly seen in patients with IBS. Faecal microbiota transplantation (FMT) has been described as an emerging, effective treatment for IBS. To date, there have been no reports on changes in food intolerances in patients with IBS treated with FMT. A 35-year-old female presented with an 18-month history of nausea, abdominal pain, alternating bowel habits, headaches/migraines, brain fog, and fatigue. The patient described severe food intolerances to dairy, gluten, egg, and soy. Extensive investigation identified no pathology, and IBS was diagnosed. The patient underwent treatment with antibiotics followed by treatment with FMT enema infusions decreasing in frequency for 12 months. At 12-week follow-up during FMT infusions, the patient reported an $80-90 \%$ improvement in her symptoms with formed bowel motions 1-2/day, occasional abdominal pain, and nausea and improved energy levels. The patient reported progressive improvements in her food tolerance from approximately 2 months into the FMT treatment. At week 24, she was tolerating gluten and dairy foods in her diet. At week 52 , she was including gluten and dairy in her diet with no symptoms. This case demonstrates that antibiotics, followed by FMT may be an effective treatment for IBS and food intolerances pointing to the gut microbiome as a potential target for treatment.
\end{abstract}




\section{Background}

Irritable bowel syndrome (IBS) is a chronic gastrointestinal condition characterized by abdominal pain and altered bowel habits. IBS affects around $12 \%$ of the world's population and is known to significantly impact quality of life. Treatments are predominantly symptom focused, due to a limited understanding of the aetiology. Evidence suggests IBS is likely multifactorial with a strong interplay of the gut microbiome, diet, gut endocrine cells, and central nervous system. Patients with IBS commonly attribute their symptoms to a variety of foods, although the mechanisms and definitions of these food reactions are poorly defined [1,2].

Adverse food reactions are broadly classified into 2 types, immune mediated: those that involve the immune system such as immunoglobulin-E mediated food allergy or coeliac disease and non-immune mediated: those that do not-involve the immune system such as enzyme deficiency, and sensitivity to fermentable carbohydrates, wheat/gluten, biogenic amines, or food additives. Non-immune food reactions or food intolerances are thought to affect up to $20 \%$ of the general population and are commonly seen in patients with IBS. Currently, the management of food intolerances is limited to diagnostic elimination diet and challenges, with patients having to limit food triggers long term $[3,4]$. This has significant impacts on nutritional adequacy and quality of life [5].

Faecal microbiota transplantation (FMT) has been described as an emerging, effective treatment for IBS [6, 7]. FMT is the process in which homogenized, filtered stool, from a highly screened, healthy donor is infused into the bowel of an unwell recipient to provide therapeutic benefit [8]. To date, there have been no reports on improvements in food intolerances in patients with IBS treated with FMT. Therefore, this case report of improvement in food intolerance and IBS symptoms after antibiotics and FMT in a young female patient constitutes an important insight into a possible involvement of the gut microbiome in the pathogenesis of food intolerances.

\section{Case Presentation}

A 35-year-old female presented to our clinic with an 18-month history of nausea, abdominal pain, and alternating loose stools or constipation. She also reported symptoms of headaches/ migraines, brain fog, and fatigue. The patient described severe food intolerances to dairy, gluten, egg, and soy and moderate intolerances to onion/garlic, raw vegetables, and high fructose fruits, for example, apples and pears (Table 1). Medical history comprised anxiety, sessile serrated adenoma, orthostatic hypotension, iron deficiency anaemia, and vasovagal syncope. Medications included low dose Endep, Zoloft, and psyllium husks. The patient underwent extensive testing including stool tests, endoscopy, and colonoscopy with luminal culture and mucosal biopsy, urea breath test, hydrogen breath tests, abdominal CT scan, and blood tests. A small hiatus hernia and renal calculus were identified. No other pathology was identified and IBS diagnosis, with suspected gut microbiota dysbiosis was made.

Non-absorbable antibiotics are an effective treatment for IBS $[2,9]$ and have been used at our centre in conjunction with FMT since the 1980s [10,11]. Choice of antibiotic therapy is individualized by the physician dependent on the patient's clinical presentation and medical history. Patients are followed up every 4 weeks to assess response and make adjustments to the treatment as required. FMT therapy is used following antibiotic treatment response to repopulate the gut microbiota. An intensive 6-month FMT treatment has been standard at our centre since 2018 due to our experience and increasing evidence that repeated FMTs are required to obtain ongoing clinical remission $[12,13]$.

The patient was subsequently commenced on vancomycin (250 mg TDS) for suspected gut microbiota dysbiosis. At her 1-month follow-up, she reported no constipation with formed

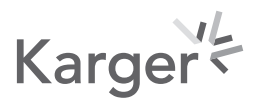


Table 1. Change in patient reported food intolerances over the course of the antibiotic and FMT treatment

\begin{tabular}{|c|c|c|c|c|c|}
\hline \multirow[t]{2}{*}{ Food } & \multirow{2}{*}{$\begin{array}{l}\text { Pretreatment } \\
\text { symptom/reaction }\end{array}$} & \multicolumn{2}{|l|}{ On antibiotics } & \multicolumn{2}{|l|}{ Week 24 FMT } \\
\hline & & symptom/reaction & change & symptom/reaction & change \\
\hline Dairy & $\begin{array}{l}\text { Migraine and } \\
\text { bloating }\end{array}$ & $\begin{array}{l}\text { Migraine and } \\
\text { bloating }\end{array}$ & Reduction & No symptoms & No symptoms \\
\hline Gluten & Bloating & Bloating & Reduction & No symptoms & No symptoms \\
\hline Egg & Nausea & Nausea & No change & Nausea & $\begin{array}{l}\text { Significant } \\
\text { reduction }\end{array}$ \\
\hline Soy & Nausea & Nausea & Reduction & Nausea & $\begin{array}{l}\text { Significant } \\
\text { reduction }\end{array}$ \\
\hline Onion/garlic & Abdominal pain & Abdominal pain & Reduction & No symptoms & No symptoms \\
\hline $\begin{array}{l}\text { Raw vegetables } \\
\text { especially leafy } \\
\text { greens }\end{array}$ & $\begin{array}{l}\text { Abdominal pain } \\
\text { and bloating }\end{array}$ & $\begin{array}{l}\text { Abdominal pain } \\
\text { and bloating }\end{array}$ & No change & $\begin{array}{l}\text { Abdominal pain } \\
\text { and bloating }\end{array}$ & Reduction \\
\hline $\begin{array}{l}\text { High fructose } \\
\text { fruit }\end{array}$ & $\begin{array}{l}\text { Abdominal pain } \\
\text { and bloating }\end{array}$ & $\begin{array}{l}\text { Abdominal pain } \\
\text { and bloating }\end{array}$ & Reduction & $\begin{array}{l}\text { Abdominal pain } \\
\text { and bloating }\end{array}$ & $\begin{array}{l}\text { Significant } \\
\text { reduction }\end{array}$ \\
\hline
\end{tabular}

FMT, Faecal microbiota transplantation.

bowel motions 3-5 daily and improvements in abdominal pain. However, symptoms of nausea and fatigue persisted. Due to her partial improvement, the patient was subsequently switched to rifaximin (500 mg TDS) and FMT planning instigated. One month after commencing rifaximin, the patient reported further improvements with formed bowel motions twice daily and a reduction in abdominal pain, bloating, nausea, and fatigue. The patient also reported small improvements in gluten and dairy intolerances.

The patient subsequently underwent FMT, with the first dose administered by colonoscopy, followed by FMT enemas in decreasing frequency from weekly to fortnightly over 12 months. The patient completed at total of 32 FMTs in the first 6 months, with 3 different donors and continued self-administering 1-2 enemas per month for the remaining 6 months. All FMT donor stool was processed according to Australian and international guidelines $[8,14]$. During the FMT treatment period, the patient also completed validated self-administered questionnaires including the abdominal symptoms questionnaire, SF-36 quality of life questionnaire [15], and IBS Birmingham questionnaire [16] on commencement of FMT and weeks 4, 12, and 24 of treatment. Three-day weighed food records were also collected at the same time-points.

Overall, the patient demonstrated continuing symptom improvement and increased QOL over the course of the FMT treatment (Fig. 1-3). There were no reported adverse events. At her 12-week follow-up appointment during FMT, she reported an 80-90\% improvement in her symptoms with formed bowel motions 1-2/day, only occasional abdominal pain and nausea and improved energy levels. At her 24-week follow-up, the patient reported continued improvement in her symptoms and also noted an overall improvement in her mental health.

The patient reported improvements in her food tolerance from approximately 2 months into the FMT treatment. She reported trialling gluten (as bread) at 2 months and was successfully able to tolerate 1 sandwich per week and increase this to consuming gluten containing foods (bread and pasta) 3-4 times per week by week 24. Dairy products (cheese and yoghurt) were also introduced at 2 months and tolerated daily. Small amounts of egg and soy were also trialled with mild symptoms of nausea (Table 1). By week 52, the patient reported no symptoms with the consumption of gluten or dairy containing foods, nor with onion and garlic. She continued to report mild symptoms with the consumption of egg, soy, raw vegetables, and high fructose fruits. 
Fig. 1. Change in SF-36 quality of life scores over 24 weeks of FMT treatment. A higher score indicates a greater quality of life. PF, physical functioning; BP, bodily pain; $\mathrm{MH}$, mental health; $\mathrm{V}$, vitality; $\mathrm{SF}$, social functioning; RLEP, role limitations due to emotional problems; $\mathrm{GH}$, general health; RLPH, role limitations due to physical health; QOL, quality of life; FMT, faecal microbiota transplantation.

Fig. 2. Change in Birmingham IBS symptom score over 24 weeks of FMT treatment. A lower score indicates a lower severity of symptoms. IBS, irritable bowel syndrome; FMT, faecal microbiota transplantation.

Fig. 3. Change in abdominal symptoms over 24 weeks of FMT treatment. A lower score indicates a lower severity of symptoms. FMT, Faecal microbiota transplantation.
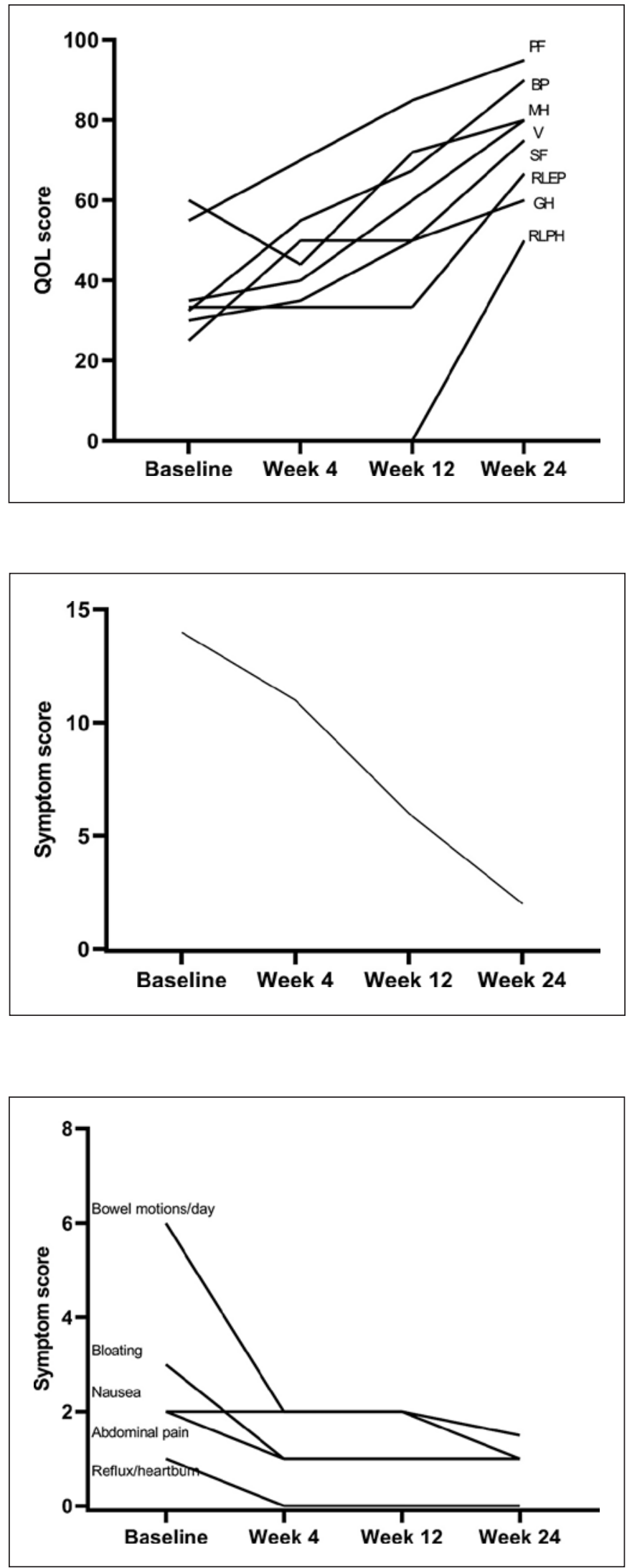


\section{Discussion and Conclusions}

This case demonstrates that antibiotics, followed by FMT can be an effective treatment for IBS and food intolerances. Continued improvement in IBS symptoms, QOL, and food intolerances was found up to 12 months after commencing FMT treatment. To the best of our knowledge, this is the first case report describing FMT improving symptoms of severe food intolerances.

A 2019 study by Kenyon et al. [17] described a retrospective cohort of 42 patients with IBS and chronic fatigue syndrome treated with FMT. Three of the patients were noted by the authors to have food sensitives prior to FMT. One patient was reported to have gradual resolution of food sensitives after FMT. Changes in food sensitivity were not described for the remaining 2 cases following FMT. Conversely, a study assessing the long-term safety and efficacy of FMT for treatment of recurrent Clostridium difficile infection noted that 1 patient reported increased sensitivity to brassica vegetables following FMT [18]. A similar study by Jalanka et al. [19] (2017) reported the development of "allergies" in 3 patients post-FMT for recurrent Clostridium difficile infection. These "allergies" included sensitivity to chemicals, birch pollen, and foods [19].

Mechanisms for the development and perpetuation of food intolerances are poorly understood and are likely variable depending on the culprit food/food component. Possible mechanisms for the development of food intolerances could include depletion of protective bacterial strains from the gut microbiota with beneficial metabolic function or the presence of infections (viral or bacterial opportunistic pathogens), resulting in a pro-inflammatory state or visceral hypersensitivity. Genetic predisposition may also influence the individual response to these environmental changes [20]. For example, a recent study has reported differences in gut microbiota composition in a small sample of patients with biogenic amine intolerances including low levels of Verrucomicrobia and non-significant increases in Proteobacteria compared to controls. Interestingly, increased levels of Proteobacteria have also been reported in patients with IBS. Elevated levels of Proteobacteria have also been suggested as a marker for epithelial dysfunction, providing a potential mechanism for histamine intolerance, in which the histamine degrading Diamine Oxidase enzyme is decreased due to disruption in mucosal epithelial cells where it is usually stored [21]. Similarly, the proposed mechanism of non-coeliac gluten sensitivity involves pre-existing gut dysbiosis, characterized by low levels of bifidobacteria, commonly seen in patients with IBS, resulting in decreased butyrate production. Subsequently, the gut is no longer able to process certain inflammatory stimuli from gluten containing foods such as wheat $\alpha$-amylase trypsin inhibitors or microbial lipopolysaccharides [22]. Together with this case report, evidence suggests that food intolerances are likely a consequence of IBS, may be mediated by the gut microbiota and thus modulation by antibiotics, and FMT may improve food tolerance. Future studies should profile the gut microbiota of patients with food intolerances and the changes following treatment.

Strengths of this case report included use of prospectively collected, objective, validated questionnaires to quantify symptom improvement during FMT treatment. Food intolerances were also well described including type of foods and change in symptoms. However, the limitations of this case report are also recognized including the retrospective nature of data collection, inability to generalize and identify a true-cause, and effect relationship. Future research should focus on prospective clinical trials of FMT treatment in IBS with the inclusion of documentation of well-defined food sensitivities. Overall, this case demonstrates that antibiotics, followed by FMT can be an effective treatment for IBS and food intolerances.

\section{Acknowledgements}

The authors would like to acknowledge the contributions of the participant to the content of the manuscript.

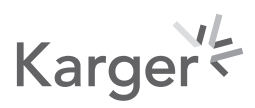


Clancy and Borody: Antibiotics and Faecal Transplant Improve Food Intolerance

\section{Statement of Ethics}

Ethics approval and consent to participate: This study was approved by the institutional human research Ethics Committee (CDD20C01). The patient provided written informed consent for the publication of this case report.

\section{Conflict of Interest Statement}

T.B. has a pecuniary interest in the Centre for Digestive Diseases, is a medical advisor to Finch therapeutics, and holds patents in FMT treatment. A.C. has no disclosures.

\section{Funding Sources}

No specific funding was received for this manuscript.

\section{Author Contributions}

Annabel Clancy collated and analysed the data and drafted the manuscript. Thomas Borody was involved in the study conception, patient management, and drafting of the manuscript. All the authors read and approved the final manuscript.

\section{Availability of Data and Material}

All data generated or analysed during this study are included in this published article (and its supplementary information files).

\section{References}

1 El-Salhy M, Hatlebakk JG, Hausken T. Diet in irritable bowel syndrome (IBS): interaction with gut microbiota and gut hormones. Nutrients. 2019;11(8):1-15.

2 Chong PP, Chin VK, Looi CY, Wong WF, Madhavan P, Yong VC. The microbiome and irritable bowel syndrome: a review on the pathophysiology, current research and future therapy. Front Microbiol. 2019;10(JUN): $1-23$.

3 Tuck CJ, Biesiekierski JR, Schmid-Grendelmeier P, Pohl D. Food intolerances. Nutrients. 2019;11(7):1-16.

4 Turnbull JL, Adams HN, Gorard DA. Review article: the diagnosis and management of food allergy and food intolerances. Aliment Pharmacol Ther. 2015;41(1):3-25.

5 Staudacher HM. Nutritional, microbiological and psychosocial implications of the low FODMAP diet. J Gastroenterol Hepatol. 2017;32(Suppl 1):16-9.

6 Holvoet T, Joossens M, Vázquez-Castellanos JF, Christiaens E, Heyerick L, Boelens J, et al. Fecal microbiota transplantation reduces symptoms in some patients with irritable bowel syndrome with predominant abdominal bloating: short- and long-term results from a placebo-controlled randomized trial. Gastroenterology. 2021;160(1):145-e8.

7 Johnsen PH, Hilpüsch F, Valle PC, Goll R. The effect of fecal microbiota transplantation on IBS related quality of life and fatigue in moderate to severe non-constipated irritable bowel: secondary endpoints of a double blind, randomized, placebo-controlled trial. EBioMedicine. 2020;51:102562.

8 Haifer C, Kelly CR, Paramsothy S, Andresen D, Papanicolas LE, McKew GL, et al. Australian consensus statements for the regulation, production and use of faecal microbiota transplantation in clinical practice. Gut. 2020;69(5):801-10.

9 Andrews CN, Sidani S, Marshall JK. Clinical management of the microbiome in irritable bowel syndrome. J Can Assoc Gastroenterol. 2021;4(1):36-43. 
10 Borody TJ, George L, Andrews P, Brandl S, Noonan S, Cole P, et al. Bowel-flora alteration: a potential cure for inflammatory bowel disease and irritable bowel syndrome? Med J Aust. 1989 May 15;150:604.

11 Borody TJ, Noonan S, Cole P, Hyland L, Morgan A, Leene J, et al. Oral vancomycin can reverse idiopathic constipation. Gastroenterology. 1989;96:52A.

12 Paramsothy S, Kamm MA, Kaakoush NO, Walsh AJ, van den Bogaerde J, Samuel D, et al. Multidonor intensive faecal microbiota transplantation for active ulcerative colitis: a randomised placebo-controlled trial. Lancet. 2017 Mar 25;389(10075):1218-28.

13 El-Salhy M, Hausken T, Hatlebakk JG. Increasing the dose and/or repeating faecal microbiota transplantation (FMT) increases the response in patients with irritable bowel syndrome (IBS). Nutrients. 2019;11(6):1415.

14 Cammarota G, Ianiro G, Tilg H, Rajilić-Stojanović M, Kump P, Satokari R, et al. European consensus conference on faecal microbiota transplantation in clinical practice. Gut. 2017;66:569-80.

15 Russell A, Ball J, Spallek M. ALSWH data dictionary supplement; 2018.

16 Roalfe AK, Roberts LM, Wilson S. Evaluation of the Birmingham IBS symptom questionnaire. BMC Gastroenterol. 2008;8:30-7.

17 Kenyon JN, Coe S, Izadi H. A retrospective outcome study of 42 patients with chronic fatigue syndrome, 30 of whom had irritable bowel syndrome. Half were treated with oral approaches, and half were treated with faecal microbiome transplantation. Hum Microbiome J. 2019;13:100061.

18 Tabbaa OM, Aboelsoud MM, Mattar MC. Long-term safety and efficacy of fecal microbiota transplantation in the treatment of clostridium difficile infection in patients with and without inflammatory bowel disease: a tertiary care center's experience. Gastroenterol Res. 2018;11(6):397-403.

19 Jalanka J, Hillamaa A, Satokari R, Mattila E, Anttila VJ, Arkkila P. The long-term effects of faecal microbiota transplantation for gastrointestinal symptoms and general health in patients with recurrent Clostridium difficile infection. Aliment Pharmacol Ther. 2018;47(3):371-9.

20 Caminero A, Meisel M, Jabri B, Verdu EF. Mechanisms by which gut microorganisms influence food sensitivities. Nat Rev Gastroenterol Hepatol. 2019;16(1):7-18.

21 Schink M, Konturek PC, Tietz E, Dieterich W, Pinzer TC, Wirtz S, et al. Microbial patterns in patients with histamine intolerance. J Physiol Pharmacol. 2018;69(4):579-93.

22 Leccioli V, Oliveri M, Romeo M, Berretta M, Rossi P. A new proposal for the pathogenic mechanism of noncoeliac/non-allergic gluten/wheat sensitivity: piecing together the puzzle of recent scientific evidence. Nutrients. 2017;9(11):1203. 